\title{
Brace treatment for adolescent idiopathic scoliosis - Protocols of the Canadian Spinal Deformity Study Group Surgeons
}

\author{
Douglas L Hill, Eric C Parent", Edmond Lou, Marc J Moreau, James K Mahood, Douglas M Hedden \\ From 7th International Conference on Conservative Management of Spinal Deformities \\ Montreal, Canada. 20-22 May 2010
}

\section{Introduction}

Rigid full-time braces are the most common non-surgical treatment for adolescents with moderate severity of scoliosis and further growth. The Scoliosis Research Society has established guidelines on which patients with adolescent idiopathic scoliosis (AIS) should be treated with a brace. This study surveyed Canadian surgeons on their protocols for prescribing braces for adolescent females with AIS.

\section{Materials and methods}

An on-line survey of 41 questions was developed to document patient profiles and surgeon protocols for prescribing braces. Recommendation on bracing was based on scenarios of three levels of maturity, six levels of curve severity, and whether or not the curve was progressive. The survey was administered between July and November 2008 to the 30 paediatric spine surgeons of the Canadian Paediatric Spinal Deformities Study Group. After one reminder, the response rate was $70 \%$ (21/30), representing 12 Canadian spine centres.

\section{Results}

The majority ( $81 \%$ ) of braces prescribed were rigid fulltime braces followed by rigid night-time braces (14\%). Average age of referral was 11-12 years for half the centres and over 12 years for the remainder. Most (81\%) centers required radiographs prior to the first clinic visit. All surgeons recommended bracing, but there was broad variation on who they considered should be braced. This high variability was also observed among surgeons in the same centre. Detection of curve progression increased the likelihood of bracing for curves $<35$ degrees. The majority (70\%) of surgeons recommend bracing within the SRS guidelines. Only progressive pre-menarchal females with 25-35 degree curves or 25-30 degree curves within 1 year of menarche had $>80 \%$ agreement on bracing. Braces were not recommended by $>50 \%$ of respondents for females with less than 1 year growth remaining regardless of progression or curve size. All considered family issues and patient acceptance when recommending a brace. Age and curve severity were criteria for bracing; skeletal maturity was the primary criteria for discontinuing bracing. Weaning was common (76\%), but protocols varied.

\section{Conclusion}

In spite of SRS guidelines and general agreement that braces are effective, there is little agreement among surgeons on which females with AIS should be treated with a brace. The likelihood that a female with AIS will be prescribed a brace primarily depends on surgeon brace prescription patterns, rather than spine curvature.

Published: 10 September 2010

doi:10.1186/1748-7161-5-S1-039

Cite this article as: Hill et al.: Brace treatment for adolescent idiopathic scoliosis - Protocols of the Canadian Spinal Deformity Study Group Surgeons. Scoliosis 2010 5(Suppl 1):O39.

University of Alberta, Edmonton, Canada

Full list of author information is available at the end of the article 